\title{
Effect of interferential current therapy versus cryotherapy on knee pain in osteoporotic postmenopausal women: a single-blind randomized controlled trial
}

\author{
DOI: https://doi.org/10.5114/pq.2019.86462
}

\author{
Shiymaa M. Abdo', Azza B. Nashed', Marwa E. Hasanin', Reda E.S. Yassin² \\ ${ }^{1}$ Department of Physical Therapy for Woman's Health, Faculty of Physical Therapy, Cairo University, Giza, Egypt \\ ${ }^{2}$ El Sahel Teaching Hospital, Cairo, Egypt
}

\begin{abstract}
Introduction. Postmenopausal knee pain, due to lack of oestrogen, is an important medical and socioeconomic problem that affects the quality of life. The most important symptom is pain during walking. The purpose of the study was to compare the effect of interferential current therapy vs. cryotherapy on knee pain in postmenopausal women.

Methods. Overall, 30 postmenopausal women with knee pain, aged 50-60 years, were assigned randomly into 2 equal groups. Group A received interferential current therapy for 4 weeks, while group B received cryotherapy for 4 weeks. General knee pain severity, knee pain severity during walking, and femoral neck bone mineral density (BMD) were assessed before and after treatment through visual analogue scale, walking pain scale, and dual-energy X-ray absorptiometry, respectively.

Results. Statistical analysis revealed a significant reduction in knee pain severity generally as well as during walking and a significant increase of femoral neck BMD after treatment in both groups $(p=0.0001)$. When comparing both groups post-treatment, there was a highly significant reduction in knee pain severity generally as well as during walking $(p=0.0001)$ in favour of group $A$, while a statistically non-significant difference was observed in the increase in femoral neck BMD between the groups $(p>0.05)$. Conclusions. Interferential current therapy is more effective than cryotherapy in reducing the severity of knee pain, both generally and during walking, in osteoporotic postmenopausal women.

Key words: interferential current therapy, cryotherapy, knee pain, osteoporosis, menopause
\end{abstract}

\section{Introduction}

Menopause is experienced by 1.5 million women each year and often involves troublesome symptoms, including vasomotor symptoms, insomnia, fatigue, and joint pain [1]. Postmenopausal women are at risk for osteoporosis, which manifest clinically as pain and physical disability [2].

Joint pain is mostly correlated with changes that take place during menopause, which affect oestrogen levels and the vasomotor system. Joint pain becomes intense, includes swelling, redness, and tenderness, and the area becomes deformed [3]. Musculoskeletal pain is more common in women than men; sex hormones, as well as psychosocial factors are related to increased perception of pain in women compared with men [1]. The prevalence of widespread knee pain is clearly related to age, with a significant increase in subjects over 50 years of age. In a population study, longstanding knee pain in women was more often part of widespread pain than in men (68\% vs. $40 \%)$ [4].

Current therapies for knee pain are directed towards pain relief and reduction of secondary functional disability. Firstly, they include physical agents such as interferential current therapy and cryotherapy [5].

Interferential therapy is characterized by the interference of 2 medium frequency currents, which are combined to produce an effective current with lower impedance to the skin and deeper penetration into tissue [6]. Interferential therapy is used for the treatment of acute and chronic pain and plays an important role in the treatment of knee pain [5].

Physiological and neurological responses to cryotherapy in musculoskeletal tissues have been extensively examined in the literature. Decreasing temperature in skin, muscle, and/or intra-articular structures results in relief in clinical problems such as acute inflammatory processes, pain, swelling, muscle spasms, and symptoms of delayed-onset muscle soreness [6]. Cryotherapy was found, however, to have detrimental effects on motor function, including nerve conduction velocity and synaptic transmission, muscle spindle sensitivity, firing rates, muscle strength, postural control, and functional performance [7].

Unfortunately, the literature review was unable to identify any study comparing the effect of interferential current therapy vs. cryotherapy on knee pain in osteoporotic postmenopausal women. Consequently, we conducted the presented study to compare their different impacts on postmenopausal knee pain in female patients.

\section{Subjects and methods}

\section{Study design}

The study was designed as a prospective, randomized, controlled trial. It was conducted between May and November 2018.

Correspondence address: Marwa E. Hasanin, Faculty of Physical Therapy, Cairo University, 7 Ahmed ELzayat St. BienElsarayat, Dokky, Giza, Egypt, e-mail: marwagynea@gmail.com

Citation: Abdo SM, Nashed AB, Hasanin ME, Yassin RES. Effect of interferential current therapy versus cryotherapy on knee pain in osteoporotic postmenopausal women: a single-blind randomized controlled trial. Physiother Quart. 2020;28(1):30-34; doi: https://doi.org/10.5114/ pq.2019.86462. 


\section{Participants}

A total of 30 postmenopausal women were recruited from the orthopaedic department of El Sahel Teaching Hospital, Cairo, Egypt. The inclusion criteria were postmenopausal osteoporosis confirmed by dual-energy $\mathrm{X}$-ray absorptiometry (DEXA) (T-score $\leq-2.5)$ and knee pain for at least 12 weeks. The patients' age ranged from 50 to 60 years and their body mass index (BMI) was up to $25 \mathrm{~kg} / \mathrm{m}^{2}$. The participants were excluded if they had other causes for knee pain (traumatic, metabolic, or neurological causes, as well as morbid obesity) or history of collagen diseases and if they used any kind of medications that could influence bone metabolism, as shown in Figure 1.

\section{Randomization}

Each participant was informed about the nature, purpose, and benefits of the study, the right to refuse or withdraw at any time, and the confidentiality of any obtained data. The postmenopausal women were randomly assigned into 2 equal groups ( $A$ and $B$ ) with the use of a computer-based randomization program. No dropping out of subjects from the study was reported after randomization. The patients were blinded to their allocation.

\section{Interventions}

Group A was composed of 15 postmenopausal women who received interferential current therapy for 4 weeks. Group B consisted of 15 postmenopausal women who received cryotherapy for 4 weeks ( 3 sessions per week for both groups).

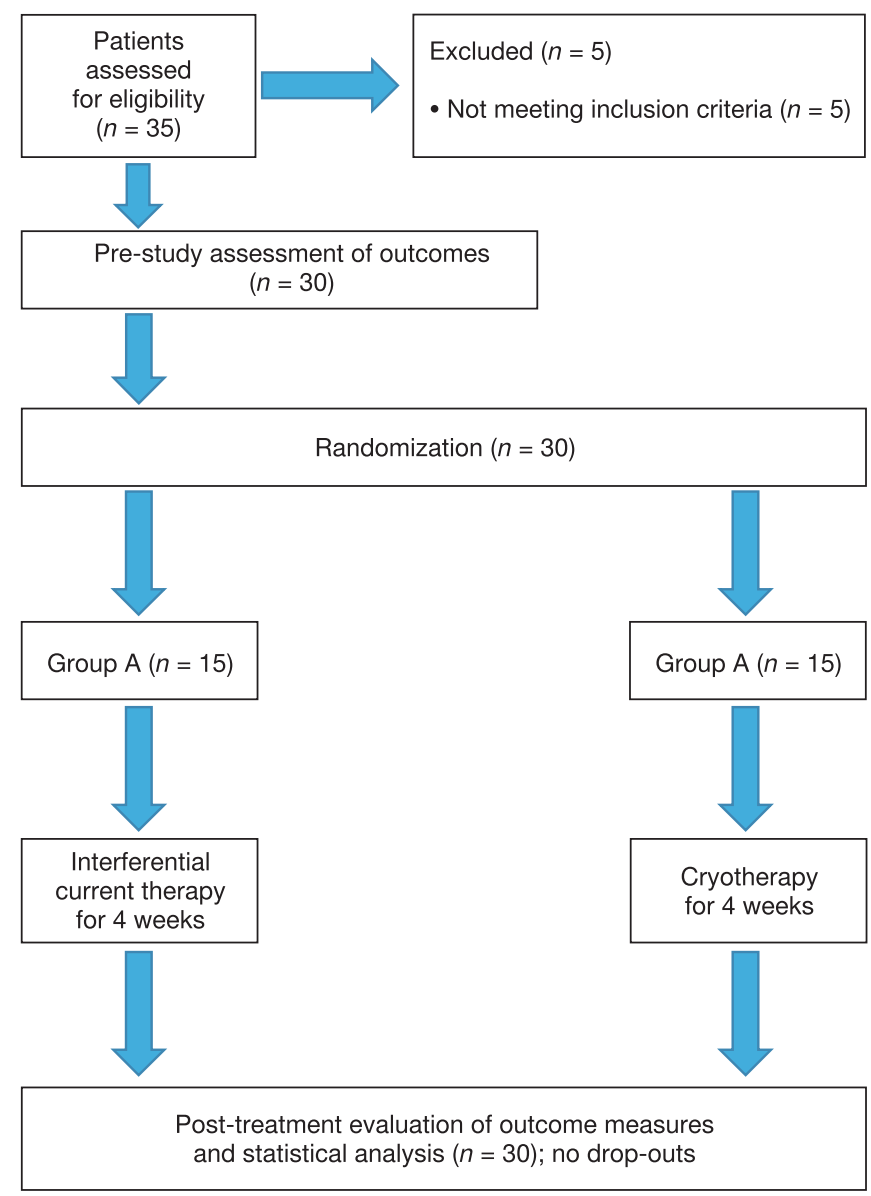

Figure 1. Flow chart of the study

\section{Instrumentation}

The following instruments were used:

- DEXA, to measure bone mineral density (BMD);

- interferential current therapy: Chattanooga Intellect Mobile Combo (ENDOMED 182, the Netherlands) with 2/4 pole interferential current;

- cryotherapy: Gymna Cryoflow ICE-CT (Uniphy Elektromedizin $\mathrm{GmbH} \&$ Co. KG, Germany).

\section{Evaluation procedure}

The evaluation procedure was performed for all patients in the 2 groups before starting the program and after 4 weeks of treatment.

\section{Pain assessment}

Pain was evaluated by using the visual analogue scale (VAS). Each subject was made aware that VAS constituted a 10-cm horizontal line with one end described as 'no pain' (0), and the other end as 'the worst pain I have ever felt' (10). The patients were asked to mark a point on the line between the extremes that related to their level of pain.

\section{Assessment of walking pain}

Walking pain was assessed with a walking pain scale. Each subject was made aware that the scale constituted a horizontal line with indications from 0 to 4 . The patients were asked to mark a point on the line between the extremes that related to their degree of pain.

\section{Assessment of bone mineral density}

DEXA was used to measure BMD before and after the course of treatment. The patient lay in the supine position without any movement and was exposed to dual-energy X-ray. The examination provided information on the presence of osteoporosis or osteopenia. It may also help identify women likely to develop osteoporosis.

On the day of the examination, the patients followed their normal diet and avoided taking any calcium supplements, wearing any garments with zippers, belts, or buttons made of metal. They were instructed to wear a gown during the examination. Any jewellery and any metal objects or clothing that might interfere with the X-ray images had to be removed. The subjects were asked to inform the physician if they had recently had a barium examination or had been injected with a contrast material for a computed tomography or radioisotope scan.

\section{Treatment procedure}

\section{Group A}

Each postmenopausal woman in group A received interferential current therapy, 3 sessions per week for 4 weeks. The patients lay in a comfortable supine position. The skin overlying the affected knee was wiped with alcohol. Two pairs of electrodes, covered with padding, were placed: two electrodes lateromedially and the other two anteroposteriorly. The four electrodes were secured with a Velcro strap around the knee joint for 20 minutes. The intensity of the stimulus was gradually increased until the women felt an appreciable sensation. The frequency was $100 \mathrm{~Hz}$ for the first 15 minutes and $80 \mathrm{~Hz}$ for the next 5 minutes [8]. 


\section{Group B}

Each postmenopausal woman in group $B$ received cryotherapy, 3 sessions per week for 4 weeks. The temperature during sessions was $16^{\circ} \mathrm{C}$. The cryotherapy device continued to work until reaching vasoconstriction. Then, the device stopped to allow the temperature to return to normal in order to provide vasodilation, causing washing out of waste products. This procedure was applied for $20 \mathrm{~min}$.

\section{Outcome measures}

\section{General knee pain severity}

It was assessed for all postmenopausal women in both groups through VAS, before and after treatment. VAS is the most widely used scale in the evaluation of pain in the clinical setting and has been reported to be sensitive and reliable [9]. The scale represents the intensity dimension by a $10-\mathrm{cm}$ horizontal line, as described above.

\section{Knee pain severity during walking}

It was assessed for all postmenopausal women in both groups through a walking pain scale, before and after treatment. The walking pain scale is a horizontal line with the following indications: 0 - no pain, 1 - mild pain, 2 - moderate pain, 3 - severe pain, and 4 - worst possible pain.

\section{Femoral neck bone mineral density}

DEXA was used to measure the femoral neck BMD for all postmenopausal women in both groups, before participation in the study to confirm their diagnosis of osteoporosis and after the end of the treatment.

\section{Statistical analysis}

Statistical analysis was conducted with the SPSS for Windows software, version 22 (SPSS Inc., Chicago, USA). Prior to final analysis, the data were screened for normality assumption, homogeneity of variance, and presence of extreme scores. This exploration was performed as a pre-requisite for parametric calculations of the analysis of difference. VAS, walking pain scale, and femoral neck BMD data were normally distributed, as assessed by the Shapiro-Wilk's test $(p>0.05)$. The Levene's test reported homogeneity of variances $(p>0.05)$ for almost dependent variables (VAS, walking pain scale, and femoral neck BMD). Accordingly, $2 \times 2$ mixed MANOVA test was used to compare the tested variables of interest at different measuring periods in both groups. The value of $p \leq 0.05$ was considered significant and $p<0.01$ was considered highly significant. The sample size was determined by the availability of participants that could be recruited within the fixed time of data collection.

\section{Ethical approval}

The research related to human use has complied with all the relevant national regulations and institutional policies, has followed the tenets of the Declaration of Helsinki, and has been approved by the institutional review board at Faculty of Physical Therapy, Cairo University (No. P.T.REC/012L002197).

\section{Informed consent}

Informed consent has been obtained from all individuals included in this study.

\section{Results}

The groups were similar at baseline $(p>0.05)$ with regard to age, weight, height, BMI, and all outcome measures (Tables 1-4).

The general knee pain severity showed a highly statistically significant reduction $(p=0.0001)$ within groups $A$ and $B$. Also, the post-treatment comparison of both groups revealed a highly statistically significant decrease in the severity of general knee pain $(p=0.0001)$ in favour of group A (Table 2$)$.

The knee pain severity during walking showed a highly statistically significant reduction $(p=0.0001)$ within groups $A$ and B. Also, the post-treatment comparison of both groups revealed a highly statistically significant decrease in knee pain severity during walking $(p=0.0001)$ in favour of group $A$ (Table 3).

The femoral neck BMD showed a highly statistically significant increase $(p=0.0001)$ within groups $A$ and $B$. However,

Table 1. Baseline characteristics of postmenopausal women in groups $A$ and $B$

\begin{tabular}{|l|c|c|c|c|c|}
\hline \multirow{2}{*}{ Characteristics } & Group A & Group B & \multicolumn{2}{|c|}{ Comparison } & \multirow{2}{*}{$S$} \\
\cline { 2 - 5 } & Mean $\pm S D$ & Mean $\pm S D$ & $t$-value & $p$-value & \\
\hline Age (years) & $52.93 \pm 14.88$ & $56.53 \pm 3.68$ & -0.909 & 0.371 & NS \\
\hline Body mass $(\mathrm{kg})$ & $65.1 \pm 4.35$ & $65.33 \pm 2.49$ & -0.18 & 0.858 & $\mathrm{NS}$ \\
\hline Height $(\mathrm{cm})$ & $163 \pm 4.3$ & $162.73 \pm 3.28$ & 0.191 & 0.85 & $\mathrm{NS}$ \\
\hline BMI $\left(\mathrm{kg} / \mathrm{m}^{2}\right)$ & $24.63 \pm 0.89$ & $25.21 \pm 1.33$ & -1.409 & 0.17 & NS \\
\hline
\end{tabular}

BMI - body mass index, $S D$ - standard deviation,

$\mathrm{S}$ - significance, NS - non-significant

Table 2. General knee pain severity by VAS in groups A and B

\begin{tabular}{|l|c|c|c|c|l|}
\hline $\begin{array}{l}\text { General } \\
\text { knee pain } \\
\text { severity } \\
\text { by VAS }\end{array}$ & Pre-test & Post-test & \multirow{2}{*}{ MD } & $\begin{array}{c}\text { Percentage } \\
\text { of change }\end{array}$ & $p$-value \\
\cline { 2 - 5 } & Mean $\pm S D$ & & $65 \%$ & $0.0001^{\mathrm{HS}}$ \\
\hline Group A & $6.86 \pm 0.74$ & $2.4 \pm 0.63$ & 4.46 & $6.29 \%$ & $0.0001^{\mathrm{HS}}$ \\
\hline Group B & $7.4 \pm 0.82$ & $3.53 \pm 0.51$ & 3.87 & 52.29 & \\
\hline$M D$ & -0.54 & -1.13 & & & \\
\hline$p$-value & $0.074^{\mathrm{NS}}$ & $0.0001^{\mathrm{HS}}$ & & & \\
\hline
\end{tabular}

NS $p>0.05$, non-significant, ${ }^{\mathrm{HS}} p<0.01$, highly significant

VAS - visual analogue scale, $S D$ - standard deviation,

$M D$ - mean difference

Table 3. Knee pain severity during walking by walking pain scale in groups $A$ and $B$

\begin{tabular}{|c|c|c|c|c|c|}
\hline \multirow{2}{*}{$\begin{array}{l}\text { Knee pain } \\
\text { severity } \\
\text { during } \\
\text { walking } \\
\text { by walking } \\
\text { pain scale }\end{array}$} & Pre-test & Post-test & \multirow{2}{*}{$M D$} & \multirow{2}{*}{$\begin{array}{l}\text { Percentage } \\
\text { of change }\end{array}$} & \multirow{2}{*}{$p$-value } \\
\hline & Mean $\pm S D$ & Mean $\pm S D$ & & & \\
\hline Group A & $3.4 \pm 0.5$ & $1.46 \pm 0.51$ & 1.94 & $57 \%$ & $0.0001^{\mathrm{HS}}$ \\
\hline Group B & $3.6 \pm 0.48$ & $2 \pm 0.1$ & 1.6 & $44 \%$ & $0.0001^{\mathrm{HS}}$ \\
\hline$M D$ & -0.2 & -0.542 & & & \\
\hline$p$-value & $0.153^{\mathrm{NS}}$ & $0.0001^{\mathrm{HS}}$ & & & \\
\hline
\end{tabular}

NS $p>0.05$, non-significant, ${ }^{\mathrm{HS}} p<0.01$, highly significant

$S D$ - standard deviation, $M D$ - mean difference 
Table 4. Femoral neck BMD in groups $A$ and $B$

\begin{tabular}{|l|c|c|c|c|l|}
\hline $\begin{array}{l}\text { Femoral } \\
\text { neck BMD } \\
\left(\mathrm{g} / \mathrm{cm}^{2}\right)\end{array}$ & Pre-test & Post-test & \multirow{2}{*}{ MD $\pm S D$} & $\begin{array}{c}\text { Percentage } \\
\text { of change }\end{array}$ & $p$-value \\
\cline { 2 - 6 } Group A & $0.73 \pm 0.09$ & $0.82 \pm 0.08$ & -0.09 & $12.32 \%$ & $0.0001^{\mathrm{HS}}$ \\
\hline Group B & $0.69 \pm 0.12$ & $0.76 \pm 0.13$ & -0.07 & $10.14 \%$ & $0.0001^{\mathrm{HS}}$ \\
\hline$M D$ & 0.04 & 0.06 & & & \\
\hline$p$-value & $0.297^{\mathrm{NS}}$ & $0.176^{\mathrm{NS}}$ & & & \\
\hline
\end{tabular}

NS $p>0.05$, non-significant, ${ }^{\mathrm{HS}} p<0.01$, highly significant

BMD - bone mineral density, $S D$ - standard deviation,

$M D$ - mean difference

the post-treatment comparison of both groups revealed a statistically non-significant difference $(p>0.05)$ (Table 4$)$.

\section{Discussion}

One of the economic and health problems affecting nearly $80 \%$ of the general population is knee pain, a leading cause of disability. It has a significant economic impact not only on lost productivity, but also on health care expenditure. Knee pain is ranked first as a cause of disability and inability to work, and is expected to affect most adults at some point during their lifetime [10].

Several modalities are used for treatment of knee pain, including interferential current therapy and cryotherapy, without clear evidence about their efficacy. There are several advantages of these techniques, as they are non-addictive, non-invasive means of analgesia that are simple to use and portable, and can provide continuous pain relief for a variety of conditions [10]. This study was conducted to compare the effect of interferential current therapy vs. cryotherapy on knee pain in osteoporotic postmenopausal women.

The results revealed a highly significant reduction in knee pain severity both generally and during walking, as well as a highly significant increase in femoral neck BMD within both groups. When comparing both groups post-treatment, a highly significant reduction was observed in knee pain severity generally as well as during walking in favour of group A, while there was a non-significant difference in femoral neck BMD.

Interferential therapy is an electrotherapy modality thought to decrease pain, increase the range of motion, and reduce oedema [11]. It provides better pain management and allows the underlying knee pain condition to be more comfortably treated with patterned muscle stimulation [12]. It is used in managing acute and chronic pain of different origin. It causes stimulation at the sensory level with higher frequencies of about $100 \mathrm{~Hz}$, which impact on pain gate mechanisms, thereby masking pain symptoms. Stimulation with lower frequencies up to $10 \mathrm{~Hz}$, at motor level intensities, can be used to activate the opioid mechanism, providing a degree of relief owing to decreased activity of the sympathetic ganglion and sympathetic nerves in cases of sympathetically-maintained pain [13].

Interferential current therapy has a greater depth because of the body tissue's better tolerance of medium-frequency currents. It could stimulate local nerve cells through blocking the transmission of pain signals or by providing the release of pain-reducing endorphins. It stimulates large diameter afferent fibres and inhibits input from small diameter afferent fibres in the substantia gelatinosa of the spinal cord [11].

It exerts a motor stimulating effect combined with analgesic one. Many authors have reported a relationship between muscle strength and increasing BMD, and attributed it to the mechanical effect of muscle contraction produced by electrical stimulation on the bone that stimulates osteoblastic deposition of bone. The deposition of bone at the stressed area has been suggested to be caused by piezoelectric force [14]. Interferential therapy also results in active hyperaemia, acceleration of lymph flow, and activation of cell functions. It enables the restoration of normal tissue reactions in addition to activation of electrolyte metabolism ( $\mathrm{Ca}, \mathrm{K}, \mathrm{Na}$ ) [6].

Raisz and Prestwood [15] investigated the effect of interferential current therapy on bone healing and concluded that there was an altering of temperature in the treated tissue and an increase in hydroxyproline, amino acids, and collagen, which led to raised calcification activity. Interferential therapy accelerated callus formation with strong mineralization and stimulation of both endosteal and periosteal callus formation [15].

Joint cryotherapy may be a safe treatment that would not inhibit the normal motor function, dynamically supported by a joint. There have been interesting findings regarding its therapeutic potential to improve functional performance in individuals $[16,17]$. Cryotherapy has been combined with muscle strength rehabilitation programs in patients with joint pathology in the lower extremity. It was observed to be an effective modality not only to reduce pain, swelling, and secondary hypoxic injury during immediate care, but also to induce analgesic effects during the early stage of rehabilitation to facilitate the initiation of active therapeutic exercise [18]. Knee joint cooling increased quadriceps activation in effusion conditions. Neurophysiological response following cold application included a reduction in nerve conduction velocity, discharge rate of receptors, and receptor sensitivity. Cryotherapy also decreases mechanoreceptor sensitivity, which helps relieve muscle spasm and muscle tone [18].

Cooling the skin over the knee joint might cause a systemic or centrally mediated change in the excitability or threshold level at the motor neuron pool. This change, in turn, could lead to more neural drive to the neuromuscular junction and facilitate motor neuron activation in knee muscles [19]. Significantly increased voluntary activation and peak knee extension torque, as well as changes in maximal voluntary contractions were found following 20-minute knee joint cryotherapy. These results show that the increase in voluntary contractions may be due to a rise in volitional activation, resulting from the facilitated motor neurons [20].

Collectively, joint cryotherapy can be a promising tool to maximize muscle activation and strength for patients with joint pathology. It is suggested to use joint cryotherapy prior to exercise to have the opportunity to utilize increased motor neuron activation that provides more neural drive to the neuromuscular junction, resulting in greater muscle strength [21].

As mentioned before, there is a relationship between muscle strength and increasing BMD, attributed to the mechanical effect of muscle contraction on the bone that stimulates osteoblastic deposition of bone. The deposition of bone at the stressed area has been suggested to be caused by piezoelectric force [14].

A total of 10 professional rugby players of the Italian national team, submitted to single daily sessions of whole body cooling for 5 consecutive days $\left(-110^{\circ} \mathrm{C}, 2 \mathrm{~min}\right)$, were compared with 10 players who completed the same training protocol without whole body cooling [22]. Bone metabolism was studied with the use of biochemical parameters. The soluble receptor activator of nuclear factor $\kappa B$ ligand (RANKL) and its decoy receptor osteoprotegerin (OPG) constitute a fundamental cytokine system connecting the immune sys- 
tem and bone metabolism in order to link pro- and anti-inflammatory balance to calcium stores. RANKL is released from osteoblasts and lymphocytes and activates osteoclasts, inducing bone resorption. Osteoclasts express RANK, specific receptor of RANKL, which induces an intracellular signal to reabsorb bone. The effect of RANKL on RANK is blocked by OPG [22]. Whole body cooling did not affect plasma RANK or RANKL concentrations in the athletes, but increased OPG, thus causing the OPG/RANKL ratio, an index of resorption-toformation balance, to grow as well. The increased osteogenic potential may have a role in post-fracture recovery, but also in the prevention of more insidious stress fractures [23].

\section{Limitations}

The study lacked follow-up of the rehabilitation program to evaluate the long-lasting effect.

\section{Conclusions}

Both interferential current therapy and cryotherapy are valuable methods in reducing knee pain severity and increasing femoral neck BMD in osteoporotic postmenopausal women. The former leads to better effects in decreasing pain severity.

\section{Disclosure statement}

No author has any financial interest or received any financial benefit from this research.

\section{Conflict of interest}

The authors state no conflict of interest.

\section{References}

1. Cohen LS, Soares CN, Vitonis AF, Otto MW, Harlow BL. Risk for new onset of depression during the menopausal transition: the Harvard study of moods and cycles. Arch Gen Psychiatry. 2006;63(4):386-390; doi: 10.1001/ archpsyc.63.4.385.

2. Kanis JA, Melton LJ $3^{\text {rd }}$, Christiansen C, Johnston CC, Khaltaev N. The diagnosis of osteoporosis. J Bone Miner Res. 1994;9(8):1137-1141; doi:10.1002/jbmr.5650090802.

3. Woods NF, Mitchell ES. Symptoms during the perimenopause: prevalence, severity, trajectory, and significance in women's lives. Am J Med. 2005;118(Suppl. 12B):14-24; doi: 10.1016/j.amjmed.2005.09.031.

4. Dennerstein L, Dudley EC, Hopper JL, Guthrie JR, Burger HG. A prospective population-based study of menopausal symptoms. Obstet Gynecol. 2000;96(3):351-358; doi: 10.1016/S0029-7844(00)00930-3.

5. Barron MC, Rubin BR. Managing osteoarthritic knee pain. J Am Osteopath Assoc. 2007;107(10 Suppl 6):ES21ES27.

6. Noble GJ, Lowe AS, Walsh DM. Interferential therapy review. Part 1. Mechanism of analgesic action and clinical usage. Phys Ther Rev. 2000;5(4):239-245; doi: 10.1179/ 108331900786166588.

7. Collins NC. Is ice right? Does cryotherapy improve outcome for acute soft tissue injury? Emerg Med J. 2008; 25(2):65-68; doi: 10.1136/emj.2007.051664.

8. Hurley DA, Minder PM, McDonough SM, Walsh DM, Moore AP, Baxter DG. Interferential therapy electrode placement technique in acute low back pain: a preliminary investigation. Arch Phys Med Rehabil. 2001;82(4): 485-493; doi: 10.1053/apmr.2001.21934. reliability of the visual analogue scale in the three major Nigerian languages. Int J Allied Health Sci Pract. 2009;7(3):13.

10. Bener A, Verjee M, Dafeeah EE, Falah O, Al-Juhaishi T, Schlogl J, et al. Psychological factors: anxiety, depression, and somatization symptoms in low back pain patients. J Pain Res. 2013;6:95-101; doi: 10.2147/JPR. S40740.

11. Jorge S, Parada CA, Ferreira SH, Tambeli CH. Interferential therapy produces antinociception during application in various models of inflammatory pain. Phys Ther. 2006;86(6):800-808; doi: 10.1093/ptj/86.6.800.

12. Atamaz FC, Durmaz B, Baydar M, Demircioglu OY, lyiyapici A, Kuran B, et al. Comparison of the efficacy of transcutaneous electrical nerve stimulation, interferential currents, and shortwave diathermy in knee osteoarthritis: a double-blind, randomized, controlled, multicenter study. Arch Phys Med Rehabil. 2012;93(5):748756; doi: 10.1016/j.apmr.2011.11.037.

13. Bae YH, Lee SM. Analgesic effects of transcutaneous electrical nerve stimulation and interferential current on experimental ischemic pain models: frequencies of $50 \mathrm{~Hz}$ and $100 \mathrm{~Hz}$. J Phys Ther Sci. 2014;26(12):1945-1948; doi: 10.1589/jpts.26.1945.

14. Khan J. Principles and practice of electrotherapy, $3^{\text {rd }}$ ed. New York: Churchill Livingstone; 1994.

15. Raisz LG, Prestwood KM. Epidemiology and pathogenesis of osteoporosis. Clin Cornerstone. 2000;2(6):17; doi: 10.1016/S1098-3597(00)90001-2.

16. Hopkins JT, Adolph JT. Effects of joint cryotherapy on lower chain function. Clin Kinesiol. 2003;57(3):42-48.

17. Hopkins JT. Knee joint effusion and cryotherapy alter lower chain kinetics and muscle activity. J Athl Train. 2006; 41(2):177-184.

18. Schmid S, Moffat M, Gutierrez GM. Effect of knee joint cooling on the electromyographic activity of lower extremity muscles during a plyometric exercise. J Electromyogr Kinesiol. 2010;20(6):1075-1081; doi: 10.1016/j.jelekin.2010.07.009.

19. Kim KM, Ingersoll CD, Hertel J. Facilitation of Hoffmann reflexes of ankle muscles in prone but not standing positions by focal ankle-joint cooling. J Sport Rehabil. 2015;24(2):130-139; doi: 10-1123/jsr.2013-0123.

20. Hopkins JT, Stencil R. Ankle cryotherapy facilitates soleus function. J Orthop Sports Phys Ther. 2002;32(12): 622-627; doi: 10.2519/jospt.2002.32.12.622.

21. Harkey MS, Gribble PA, Pietrosimone BG. Disinhibitory interventions and voluntary quadriceps activation: a systematic review. J Athl Train. 2014;49(3):411-421; doi: 10.4085/1062-6050-49.1.04.

22. Galliera E, Dogliotti G, Melegati G, Corsi Romanelli MM, Cabitza P, Banfi G. Bone remodelling biomarkers after whole body cryotherapy (WBC) in elite rugby players. Injury. 2013;44(8):1117-1121; doi: 10.1016/j.injury.2012. 08.057.

23. Banfi G, Lombardi G, Colombini A, Melegati G. Wholebody cryotherapy in athletes. Sports Med. 2010;40(6): 509-517; doi: 10.2165/11531940-000000000-00000. 